\title{
Early Characteristics of the Polarimetric Tornadic Debris Signature Associated with the 20 May 2013 Newcastle-Moore, Oklahoma, Tornado
}

\author{
MATTHEW D. W. SAARI \\ Baron Services, Inc., Huntsville, Alabama \\ Department of Atmospheric Science, University of Alabama-Huntsville, Huntsville, Alabama \\ ROBERT M. LAWTON \\ Baron Services, Inc., Huntsville, Alabama \\ CHRISTOPHER J. SCHULTZ \\ Department of Atmospheric Science, University of Alabama-Huntsville, Huntsville, Alabama \\ NASA Marshall Space Flight Center, Huntsville, Alabama \\ LAWRENCE D. CAREY \\ Department of Atmospheric Science, University of Alabama-Huntsville, Huntsville, Alabama
}

(Manuscript received 22 November 2013; review completed 24 March 2014)

\section{Introduction}

On 20 May 2013, a tornado outbreak affected portions of central and eastern Oklahoma, southwestern and central Missouri, and extreme northwestern Arkansas. According to the National Weather Service (NWS), the highest rated tornado of the day began on the northwestern side of Newcastle, Oklahoma, and proceeded through the heart of Moore, Oklahoma, before lifting about $8 \mathrm{~km}(5 \mathrm{mi})$ east of Moore (NWS 2013). This intense tornado caused severe damage, lofting large amounts of debris as it passed through heavily populated areas. A dual-polarization tornadic debris signature (DPTDS) persisted for the duration of this tornado - as observed by the nearby Weather Surveillance Radar-1988 Doppler (WSR-88D) in Twin Lakes, Oklahoma (KTLX). Interestingly, this signature existed prior to the official tornado start time, as listed in the National Climatic Data Center (NCDC) Storm Events Database and the NWS storm event page (NCDC 2013; NWS 2013), and before a visual funnel was observed. The presence of a DPTDS associated with a tornado of the magnitude seen in Moore, Oklahoma, on 20 May 2013 is unsurprising. However, this case is noteworthy because the early appearance of this signature (during the tornado's development) may provide interesting insight into the processes that were occurring during the period of uncertainty associated with the onset of surface damage attributed to the tornado (and prior to the formation of a visible funnel). Thus, the purpose of this Images of Note is to present this event and to promote discussion on the operational use of DPTDSs in tornado detection, warning, and future development of automated detection techniques within the meteorological community.

Several studies over the last decade have examined DPTDSs using polarimetric weather radars [e.g., Ryzhkov et al. 2005; Carey et al. 2008; Kumjian and Ryzhkov 2008; Schultz et al. 2012a; Bodine et al. 2013; Warning Decision Training Branch (WDTB 2013); and others], and generally are in agreement about the criteria that should be used to define a DPTDS. These studies recommend defining a DPTDS as a region with a strong velocity couplet co-located with relatively high reflectivity at horizontal polarization $\left(Z_{H}>20 \mathrm{dBZ}\right)$, low values of correlation coefficient $\left(\rho_{\mathrm{hv}}<0.7-0.8\right)$, and differential reflectivity $\left(\mathrm{Z}_{\mathrm{DR}}\right)$ near $0 \mathrm{~dB}$. Several studies also recommend the examination of a signature's context and volumetric features

Corresponding author address: Matthew D. W. Saari, Dept. of Atmospheric Science, University of Alabama-Huntsville, 320 Sparkman Drive, Huntsville, AL 35899

E-mail: matthew.saari@nsstc.uah.edu 
like vertical continuity (Carey et al. 2008; Schultz et al. 2012a,b; Bodine et al. 2013) as they can help distinguish a DPTDS from signatures arising from other sources (Ryzhkov 2007; Lemon et al. 2011; Schultz et al. 2012b).

\section{Discussion}

Data were collected from full volume scans of KTLX leading up to 1956 UTC, the officially published time at which surface damage attributable to the tornado began to occur (NCDC 2013; NWS 2013). The storm in question had a classic supercell structure with a well-defined hook echo on its southwestern flank (Fig. 1a, and in a wider view at 1951 UTC in Fig. 2a). The DPTDS associated with this storm was within approximately $38 \mathrm{~km}(20.5 \mathrm{n} \mathrm{mi})$ of the KTLX site during the times of interest (1942-1955 UTC).

Figure 1a shows a close-up view of the hook echo at 1942 UTC (column 1), 1946 UTC (column 2), 1951 UTC (column 3), and 1955 UTC (column 4), displaying $Z_{H}$ (row 1 ), dealiased base velocity (row 2 ), $\rho_{\mathrm{hv}}$ (row 3 ), and $Z_{\mathrm{DR}}$ (row 4 ). There is some weak rotation evident at 1942 UTC, which strengthens significantly by 1951 UTC with a gate-to-gate velocity difference in azimuth of $29.5 \mathrm{~m} \mathrm{~s}^{-1}(57.3 \mathrm{kt})$. The $\rho_{\mathrm{hv}}$ collocated with the rotation is depressed to or below 0.8 at 1942 UTC, but drops to near 0.5 by 1946 UTC, and decreases to as low as 0.35 at 1951 UTC and 0.24 at 1955 UTC. $Z_{H}$ maxima collocated with the rotation, and $\rho_{\mathrm{hv}}$ of $<0.8$, are consistently above the defined thresholds and increase from $24 \mathrm{dBZ}$ at $1942 \mathrm{UTC}$ to $47.5 \mathrm{dBZ}$ at 1955 UTC. Finally, $Z_{\mathrm{DR}}$ in this region ranges from -3 to $+3 \mathrm{~dB}$ at 1942 UTC and $1946 \mathrm{UTC}$, but is mainly near or below $0 \mathrm{~dB}$ at $1951 \mathrm{UTC}$ and 1955 UTC. Figures $2 \mathrm{~b}-\mathrm{d}$ show volume extractions of $Z_{H}$, shear rate (see figure caption), and $\rho_{\mathrm{hv}}$, respectively, from the lowest four tilts $\left(0.5^{\circ}, 0.9^{\circ}, 1.4^{\circ}\right.$, and $\left.1.8^{\circ}\right)$ of the 1951 UTC scan. These data were extracted from the center of rotation at each tilt and clearly illustrate the vertical extent of the signature. Scatter plots of $Z_{H}$ versus $\rho_{\mathrm{hv}}$ (Fig. 2e), $Z_{H}$ versus $Z_{\mathrm{DR}}$ (Fig. 2f), and $Z_{\mathrm{DR}}$ versus $\rho_{\mathrm{hv}}$ (Fig. 2g) show individual bins consistent with a DPTDS. Although $Z_{H}$ does not reach the Ryzhkov et al. (2005) threshold at 1942 UTC and 1946 UTC, it does meet the minimum for WDTB (at 1942 UTC and 1946 UTC) and Schultz et al. (2012a; at 1946 UTC). Although $Z_{H}$ and $\rho_{\mathrm{hv}}$ meet the criteria at 1942 UTC, the rotation is minimal, and the signature lacks vertical continuity. However, it could represent the first appearance, or development, of a DPTDS. Considering the thresholds, as well as contextual features (e.g., velocity signature, hook echo development), it can be argued that a DPTDS existed during each volume time leading up to the visually confirmed tornado, at least from 1946 to 1955 UTC, and possibly as early as 1942 UTC.

From Fig. 1b, it is evident that debris was being lofted prior to the tornado starting point (NCDC 2013). Moreover, time-stamped video, as well as commentary in the video, indicate that material was being lofted into the storm prior to the formation of a visible condensation funnel (see www.youtube.com/watch? $\mathrm{v}=\mathrm{m} 18 \mathrm{Yu}$ RxsdA8\&safe=active at 07:05 into the video). Initial damage surveys did note EF0 to EF1 damage-mainly to trees-prior to 1956 UTC that was related both temporally and spatially with the first appearances of the DPTDS (NWS 2014; also see mesonet.agron. iastate.edu/wx/afos/p.php?pil=PNSOUN\&e=20130521 1950). However, the survey team attributed that damage to thunderstorm winds because the damage characteristics appeared to be inconsistent with tornado damage (G. Garfield 2013, personal communication). The authors stress that we are not necessarily advocating that the beginning point of the track be moved to the location of the first signs of the DPTDS, because extensive surveys were done on the ground, in the air, and from satellite on this particular tornado. Instead, we present a few hypothetical possibilities to the cause of the DPTDS. First, it could have been a tornado that lacked a condensation funnel because the initial damage survey and the locations of the DPTDSs are in such good agreement. Second, the observed damage could have been caused by thunderstorm straight-line winds, resulting in the lofting of light debris by the updraft. Third, the DPTDS also could have been associated with a convective scale vortex-such as those described in Tanamachi et al. (2013) — which may have aided in the lofting of light debris. Whereas any of these explanations are plausible, the available WSR-88D observations are not of sufficient resolution to fully investigate the small-scale velocity features that would help ascertain the exact cause of the damage and the resulting DPTDS.

Regardless of the exact cause of the DPTDS (whether it is attributable to tornadic or nontornadic damage), the decreasing $\rho_{\mathrm{hv}}$ and increasing shear within the signature, along with its temporal and vertical continuity, could lead the warning forecaster to believe that a damage-producing tornado had developed, especially in the absence of visual confirmation in the signature's vicinity. Moreover, 


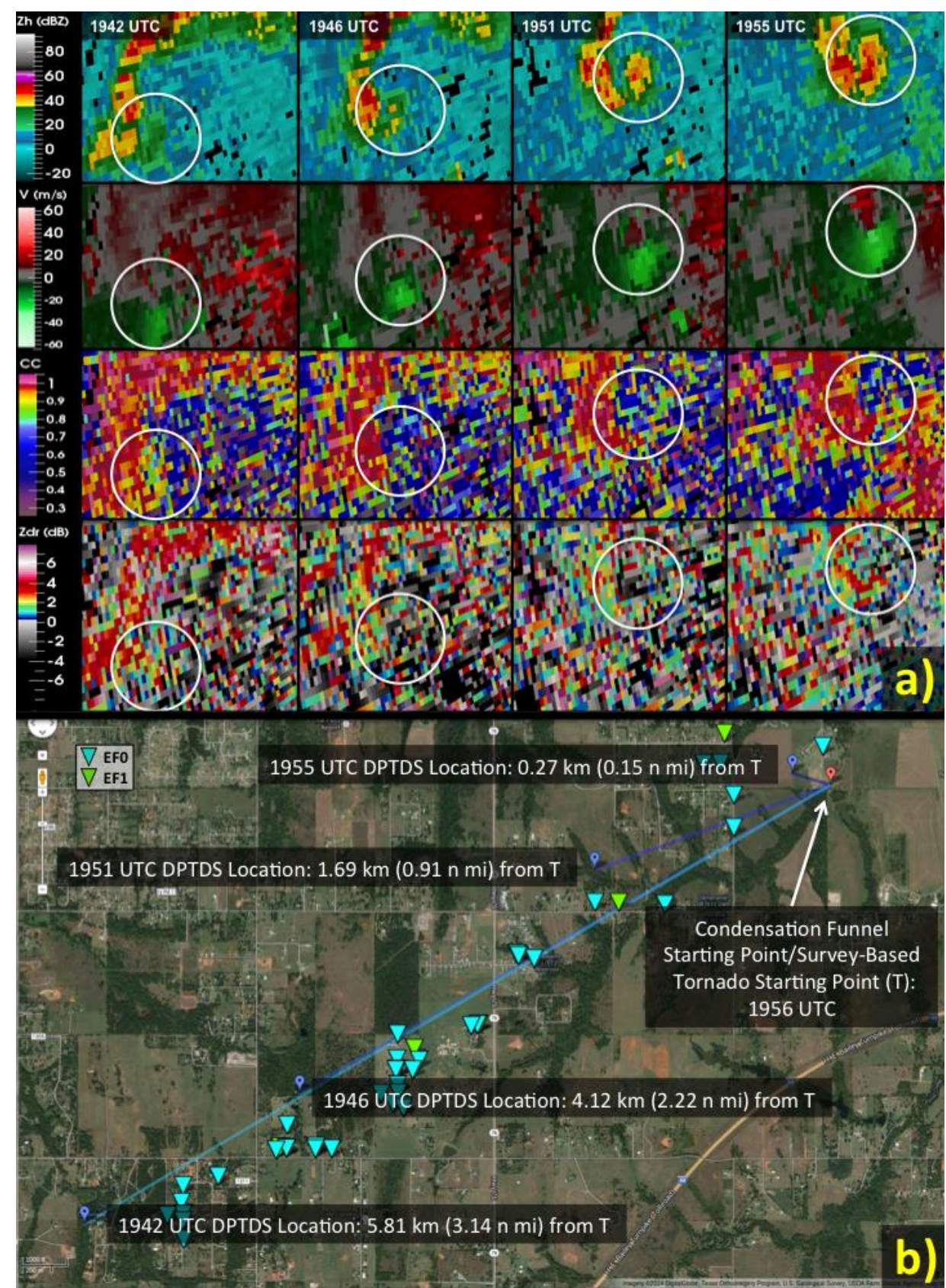

Figure 1. The top panel (a) shows a time series of the DPTDS feature prior to the visual confirmation of the condensation funnel of the Newcastle-Moore, OK, tornado of 20 May 2013 from KTLX. The DPTDS is outlined by the white circle at $0.5^{\circ}$ elevation at 1942 UTC (column 1), 1946 UTC (column 2), 1951 UTC (column 3), and 1955 UTC (column 4). Reflectivity (row 1), dealiased base velocity (row 2), correlation coefficient (row 3), and differential reflectivity (row 4) are shown for each time step. The center of each white circle represents the center of the velocity couplet at that volume time. Beam heights at the center of the white circle at 1942 through 1955 UTC are $0.43 \mathrm{~km}, 0.40 \mathrm{~km}, 0.39 \mathrm{~km}$, and $0.37 \mathrm{~km}$ AGL, respectively. The spatial scale of each box is $17.3 \mathrm{~km}(9.34 \mathrm{n} \mathrm{mi})$ in the $x$ direction, and $8.7 \mathrm{~km}(4.7 \mathrm{n} \mathrm{mi})$ in the $y$ direction. The bottom panel (b) depicts the locations of the DPTDSs at 1942-1955 UTC with respect to the tornado starting location - according to the NCDC (2013) archive. Inverted triangles depict locations of noted damage by the storm survey team, with light blue denoting EF0 equivalent damage, and green denoting EF1 equivalent damage. Map can be found online at maps.google.com/ $\underline{\mathrm{maps}} / \mathrm{ms}$ ? $\mathrm{ie}=\mathrm{UTF} \& \mathrm{msa}=0 \& \mathrm{msid}=200027741969778902130.0004 \mathrm{f} 34 \mathrm{ffb} 48 \mathrm{ac} 36 \mathrm{~d} 225 \mathrm{e}$. Click image for an external version. 


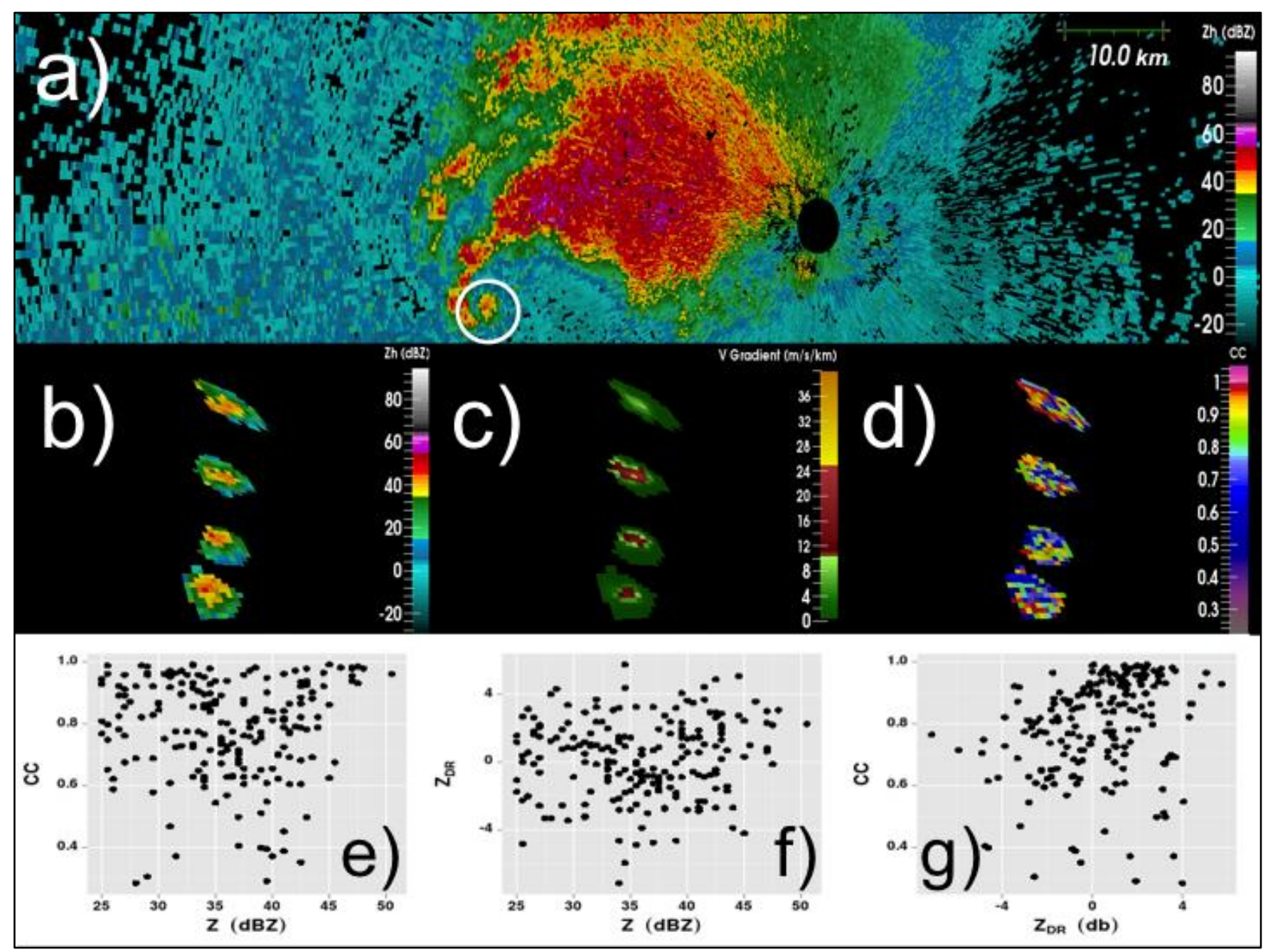

Figure 2. Examination of the DPTDS at 1951 UTC from KTLX on 20 May 2013. Panel (a) is a supercell-wide view of reflectivity at $0.5^{\circ}$ elevation. The center of the white circle denotes the location of the DPTDS at 0.39 km AGL. (b) Reflectivity, (c) shear rate [an estimate of shear with units of $\mathrm{m} \mathrm{s}^{-1} \mathrm{~km}^{-1}$, derived similar to the local, linear least-squares derivatives method discussed in Newman et al. (2013)], and (d) correlation coefficient - extracted from the DPTDS column as determined by the velocity couplet at each tilt using the $0.5^{\circ}(0.39 \mathrm{~km}$ AGL), $0.9^{\circ}(0.6 \mathrm{~km} \mathrm{AGL}), 1.4^{\circ}(0.86 \mathrm{~km} \mathrm{AGL})$, and $1.8^{\circ}(1.14 \mathrm{~km}$ AGL) degree tilts - are shown to display the vertical extent of the signature. The vertical scale in (b), (c), and (d) is expanded so that each tilt is clearly visible. The bottom row shows scatter plots of (e) reflectivity versus correlation coefficient, (f) reflectivity versus differential reflectivity, and (g) differential reflectivity versus correlation coefficient. Click image for an external version.

future radar algorithms designed for the detection of DPTDSs, especially those based solely on thresholds, likely would raise an alarm in the case presented in this note-alerting a forecaster of a likely DPTDS. However, the uncertainties and caveats presented in this note and other articles must be considered when developing such algorithms and analyzing the output they create.

\section{Summary}

By standard threshold definitions, several radar volumes leading up to the visible confirmation of the funnel associated with the 20 May 2013 NewcastleMoore tornado contained clear DPTDSs that displayed vertical continuity and were collocated with the dramatic hook echo of a well-formed supercell storm, and sporadic EF0/EF1 damage at the surface. Although we cannot be certain about the exact cause of these signatures, this event is a clear addition to the mounting body of evidence indicating that DPTDSs can and do occur in the absence of visible condensation funnels and, in some cases, the absence of substantial surface damage-likely due to the lofting of light debris (e.g., Johnstone et al. 2012; Schultz et al. 2012b). Because $\mathrm{EF} 0 / \mathrm{EF} 1$ damage does not always imply a tornado 
(e.g., Fujita and Wakimoto 1981), this article cannot resolve the current debate regarding the nature of the damage prior to the official tornado beginning point (i.e., tornadic versus nontornadic thunderstorm winds). However, what this article does is place the DPTDS observations within a framework for future discussion on the event as other data become available. Furthermore, with the entire NWS dual-polarization upgrade complete, DPTDSs without visually confirmed tornadoes in real-time will become much more common. Therefore, what implications would an event like this have on the use of automated algorithms or dissemination of a tornado threat in real-time, specifically if the event is outside of such a robust network of observation platforms and storm spotters? Finally, this event provides additional context to the current discussion of the utilization of radar data to aid in post-event storm surveys, particularly in regard to the use of polarimetric measurements in tornado verification.

Acknowledgments. We thank Rick Smith and Gabe Garfield from the Norman NWS for their review of the article and confirmation of the survey information. Thanks also to Brian Carcione and David Nadler of the Huntsville NWS for insight into how supposed DPTDSs may be used during real-time tornado operations and for enlightening discussions about precautions appropriate to the examination of apparent DPTDSs. We also thank Chris Darden (NWS Huntsville) and Steve Nelson (NWS Peachtree City) for their review and insightful comments on this article. Finally, the authors thank Mr. James LaDue, Dr. Adam Clark, and one anonymous reviewer for their insightful and constructive comments that greatly contributed to the development of this article.

\section{REFERENCES}

Bodine, D. J., M. R. Kumjian, R. D. Palmer, P. L. Heinselman, and A. V. Ryzhkov, 2013: Tornado damage estimation using polarimetric radar. Wea. Forecasting, 28, 139-158, CrossRef.

Carey, L. D., W. A. Petersen, and K. R. Knupp, 2008: ARMOR dual-polarimetric radar observations of two tornadic supercells during the 2008 Super-Tuesday tornado outbreak. Preprints, 33rd Annual Meeting, Louisville, KY, Natl. Wea. Assoc. [Available online at www.nwas.org/meetings/nwa2008/.]

Fujita, T. T., and R. M. Wakimoto, 1981: Five scales of airflow associated with a series of downbursts on 16 July 1980. Mon. Wea. Rev., 109, 1438-1456, CrossRef.

Johnstone, T., S. E. Nelson, L. Belanger, M. E. Anderson, K. B. Laws, B. Carcione, C. J. Schultz, and L. D.
Carey, 2012: Operational use of polarimetric tornadic debris signatures. Preprints, 26th Conf. on Severe Local Storms, Nashville, TN, Amer. Meteor. Soc., 16.5. [Available online at ams.confex.com/ams/26SLS/ webprogram/Paper211567.html.]

Kumjian, M. R., and A. V. Ryzhkov, 2008: Polarimetric signatures in supercell thunderstorms. J. Appl. Meteor. Climatol., 47, 1940-1961, CrossRef.

Lemon, L. R., C. A. Van Den Broeke, C. Payne, and P. T. Schlatter, 2011: Dual-polarimetric Doppler radar debris signature characteristics from a long-track EF-5 tornado. Preprints, 36th Annual Meeting, Birmingham, AL, Natl. Wea. Assoc. [Available online at www.nwas.org/ meetings/nwa2011/.]

NCDC, cited 2013: Storm events database. [Available online at www.ncdc.noaa.gov/stormevents/eventdetails.jsp ?id=451537.]

NWS, cited 2013: The tornado outbreak of May 20, 2013. [Available online at www.srh.noaa.gov/oun/?n=events20130520.]

, cited 2014: Storm damage survey. [Available online at maps.google.com/maps?q=http://en.wikipedia.org/w/ index.php\%3Ftitle\%3DTemplate:Attached_KML/2013 _Moore_tornado\%26action\%3Draw.]

Newman, J. F., V. Lakshmanan, P. L. Heinselman, M. B. Richman, and T. M. Smith, 2013: Range-correcting azimuthal shear in Doppler radar data. Wea. Forecasting, 28, 194-211, CrossRef.

Ryzhkov, A. V., 2007: The impact of beam broadening on the quality of radar polarimetric data. J. Atmos. Oceanic Technol., 24, 729-744, CrossRef.

, T. J. Schuur, D. W. Burgess, D. S. Zrnic, 2005: Polarimetric tornado detection. J. Appl. Meteor., 44, 557570, CrossRef.

Schultz, C. J., and Coauthors, 2012a: Dual-polarization tornadic debris signatures Part I: Examples and utility in an operational setting. Electronic J. Operational Meteor., 13 (9), 120-137. [Available online at www. nwas.org/ej/pdf/2012-EJ9.pdf.] , and _ 2012b: Dual-polarization tornadic debris signatures Part II: Comparisons and caveats. Electronic J. Operational Meteor., 13 (10), 138-150. [Available online at www.nwas.org/ej/pdf/2012-EJ10.pdf.]

Tanamachi, R. L., H. B. Bluestein, M. Xue, W.-C. Lee, K. A. Orzel, S. J. Frasier, and R. M. Wakimoto, 2013: Near-surface vortex structure in a tornado and in a subtornado-strength convective-storm vortex observed by a mobile, W-band radar during VORTEX2. Mon. Wea. Rev., 141, 3661-3690, CrossRef.

WDTB, cited 2013: Dual-polarization radar operations course: Tornadic debris signatures. [Available online at wdtb.noaa.gov/Training/course/index.asp?courseList2= DP_Ops\&catalogButton3=Find+Training.] 